\title{
Best Achievements in Translational and Basic Thyroidology in 2020
}

\author{
Sun Wook Cho ${ }^{1}$, Young Joo Park ${ }^{1,2}$ \\ ${ }^{1}$ Department of Internal Medicine, Seoul National University Hospital, Seoul National University College of Medicine; \\ ${ }^{2}$ Department of Molecular Medicine and Biopharmaceutical Sciences, Graduate School of Convergence Science and \\ Technology, and College of Medicine or College of Pharmacy, Seoul National University, Seoul, Korea
}

This review discusses articles published in 2020 that presented noteworthy achievements in translational and basic thyroidology. Previously unresolved questions about thyroid hormone receptor actions and signaling mechanisms were answered using various novel in vitro and in vivo models. Using high resolution cryo-electron microscopy, the fine functional structure of thyroglobulin was demonstrated, and new insights into the pathogenesis of thyroid disease were achieved, with a focus on research into thyroid-disrupting chemicals and the gut microbiome. Novel therapeutic approaches were tried in the field of advanced thyroid cancer treatments.

Keywords: Receptors, thyroid hormone; Thyroglobulin; Gastrointestinal microbiome; Thyroid neoplasm; Endocrine disruptors

\section{INTRODUCTION}

In 2020, some noteworthy advances were made in the field of basic and translational thyroidology. This review summarizes the most important research published in 2020. Peer-reviewed original research and review articles published between January 2020 and December 2020 were searched through an independent literature review. A brief summary of these articles is presented.

\section{NEW UNDERSTANDINGS OF THYROID HORMONE ACTIONS AND SIGNALING MECHANISMS}

\section{Thyroid hormone receptor alpha mutations}

Two intriguing studies using novel models of thyroid hormone receptor alpha (THRA) mutations, which cause thyroid hormone

Received: 21 January 2021, Revised: 27 January 2021, Accepted: 31 January 2021

Corresponding author: Young Joo Park

Department of Internal Medicine, Seoul National University College of

Medicine, 101 Daehak-ro, Jongno-gu, Seoul 03080, Korea

Tel: +82-2-2072-4183, Fax: +82-2-762-2292, E-mail: yjparkmd@snu.ac.kr resistance syndrome (RTH), were published in 2020. Krieger et al. [1] demonstrated the essential actions of thyroid hormone receptor alpha (TR $\alpha)$ on the normal development of the human cerebral cortex. The authors previously reported that adult patients with RTH had intellectual impairments, especially affecting nonverbal IQ and delayed sensorimotor processing [2]. Indeed, these findings were consistent with those of a mouse model with a mutant variant of TR $\alpha 1$, which showed a range of central nervous system abnormalities [3], but the cellular mechanisms of the impacts of THRA (the gene encoding TR $\alpha$ ) on neural development remained unclear. Krieger et al. [1], using THRA mutant patient-derived induced pluripotent stem cell (iPS) models in combination with a quantitative lineage analysis, gene expression analysis, and novel assays of neuro-epithelium formation, showed that THRA regulates the balance between progenitor self-renewal and neurogenesis, thereby affecting the overall brain mass and especially the cortex. A structural
Copyright $(2021$ Korean Endocrine Society

This is an Open Access article distributed under the terms of the Creative Commons Attribution Non-Commercial License (https://creativecommons.org/ licenses/by-nc/4.0/) which permits unrestricted non-commercial use, distribution, and reproduction in any medium, provided the original work is properly cited. 
modeling study of the ligand-binding domain of TR $\alpha 1$ demonstrated that the THRA mutation facilitates corepressor binding. Finally, a magnetic resonance imaging study of four patients harboring THRA mutations showed microcephaly with reduced cerebellar size and white matter density of the whole brain. Additionally, the authors suggested that their experimental method using patient-derived iPS is a good example of quantitative research in neurodevelopmental disorders.

Another study was published using a novel zebrafish model of RTH [4], which was generated by introducing dominant-negative germline mutations in both THRA alleles by the Clustered Regularly Interspaced Short Palindromic Repeats (CRISPR)/ Cas9 targeting system. Abnormalities in the heart system were observed at 35 days post-fertilization, in the juvenile period. Both anatomical abnormalities, including atrial dilatation and abnormal ventricular structures, and functional deficits such as reduced blood flow and reduced strength of contractions were observed. The key finding of this study was that the THRA mutation disrupted sarcomere organization, which gives cardiac muscles their striated appearance and critically affects heart function. The expression of genes associated with sarcomere assembly was suppressed, and structural abnormalities of sarcomere organization were found via transmission electron microscopy in the hearts of the mutant fish. This study provides an advanced understanding into the pathophysiology of bradycardia in RTH patients.

\section{Iodothyronine deiodinases}

In the research field of iodothyronine deiodinase (Dio) signaling, an interesting study was published by Ferdous et al. [5]. In cardiomyocytes, they found that forkhead box O (FoxO) 1, not FoxO3, regulates the reciprocal regulation between Dio2 and Dio3, and that FoxO1 directly upregulates Dio2 genes and positively supports $T H R A$-mediated hypertrophic growth of myocytes in neonatal rat ventricle. More importantly, FoxO1-Dio2 signaling plays an essential role in afterload-induced pathologic myocardial remodeling and activation of THRA in a transverse aortic restriction model of stress-induced cardiomyotrophy. This study highlights the importance of intracellular thyroid hormone homeostasis in the cellular response to pathologic stress.

A novel Diol mutation was reported by Franca et al. [6]. Two unrelated families had two missense pathogenic variants, resulting in p.Asn94Lys and p.Met201Ile, and showed abnormal thyroid hormone metabolism with elevated serum reverse triiodothyronine (rT3) levels and rT3/T3 ratios. This is the first report of an inherited Dio1 mutation in humans.

\section{MECHANISMS OF THYROID HORMONE- DEPENDENT TRANSCRIPTION: A GENETIC STUDY}

Recent genetic studies made great achievements in clarifying the genetic basis of thyroid function through genome-wide association studies and revealed novel pathways of the regulatory mechanisms of thyroid hormone signaling [7]. TR-dependent gene transcription was traditionally explained by a bimodal switch model, meaning that TR directly binds to DNA. Histone hyperacetylation, a hormone-dependent mechanism of activating enhancers, is also explained by a bimodal switch model, but it has not been verified by a genomic approach. Praestholm et al. [8] performed a genome-wide analysis of hyperacetylated lysine residues, $\mathrm{H} 3 \mathrm{~K} 9$ and $\mathrm{H} 3 \mathrm{~K} 27$, in murine livers from hypothyroid and hyperthyroid wild-type, TR-deficient, and nuclear receptor co-repressor $1-$ deficient mice. The authors summarized three types of thyroid hormone-regulated enhancers, and demonstrated various mechanisms modulating hormone-dependent gene transcription, thereby improving our detailed understanding of the bimodal switch model.

\section{THE STRUCTURE OF HUMAN THYROGLOBULIN}

Thyroglobulin is the protein precursor of thyroid hormones. Thyroid hormone synthesis from thyroglobulin involves a sequential process via iodination, coupling of pairs of tyrosine molecules, and finally thyroglobulin proteolysis in the thyroid gland. The proximity of tyrosine within thyroglobulin is assumed to play a pivotal role in the coupling reactions, but the hormonogenic tyrosine residues have yet to be determined. An elaborate three-dimensional structure of thyroglobulin is needed to improve the mechanistic understanding of this protein. By using high-resolution cryo-electron microscopy, Coscia et al. [9] demonstrated the full-length structure of human thyroglobulin at a resolution of $3.5 \AA$. All hormonogenic tyrosine pairs were identified and their functions were verified with cellular experiments in vitro. This study provides a valuable framework for future research into the production and regulation of thyroid hormones.

\section{GUT MICROBIOME AND THYROID DISEASE}

The health impacts of the gut microbiome in various aspects of 
human pathophysiology have been extensively studied. However, studies of the gut microbiome in thyroid disease are still in their early stage. In 2020, the thyroid-gut axis was reviewed by two groups of authors [10,11]. Briefly, dysbiosis (the disruption of gut microbiome homeostasis) is associated with autoimmune thyroid disease and several mechanisms have been demonstrated. One is direct regulation of the immune system. Dysbiosis alters intestinal barrier integrity and the resultant release of microbially-produced immunomodulatory metabolites leads to the onset of autoimmune disease with the breakdown of host immune tolerance. Another mechanism is that dysbiosis affects the availability of micronutrients including iron, iodine, selenium, and zinc.

Several basic and translational studies including animal models were published in 2020, mostly focusing on Graves' disease, which induces hyperthyroidism and is the most prevalent autoimmune endocrine disease [12]. In Graves' disease, alterations of the proportions of regulatory $\mathrm{T}$ (Treg) cells and Th17 cells, a subset of $\mathrm{T}$ helper cells producing proinflammatory interleukin 17 , have been reported [12,13]. Su et al. [14] demonstrated that gut dysbiosis plays a role in dysregulating the host immune system, including Treg/Th17 imbalance, and contributes to the pathogenesis of the activation of Graves' disease. Furthermore, that study validated the therapeutic potential of microecological treatment using Bacteroides fragilis in an animal model of Graves' disease and provided valuable clues for improving immune dysfunction in autoimmune thyroid disease. Jiang et al. [15] intensively studied the components and the metabolic features of microbiota involved in Graves' disease and generated a diagnostic model using the top nine genera distinct from those of healthy controls with an area under the curve of 0.8109 . Additionally, using an antithyroid drug-treated rat model, Sun et al. [16] demonstrated that the antithyroid drug altered the gut microbiota and destroyed the intestinal barrier, resulting in liver injury in association with increased serum lipopolysaccharide levels and related pathways.

\section{ENVIRONMENTAL FACTORS: THYROID- DISRUPTING CHEMICALS}

In recent years, the number of studies investigating the presence in the environment of chemical compounds with thyroid-disrupting effects has gradually increased. Chemicals that can affect thyroid hormone signaling during pregnancy are an important issue in this field [17], since thyroid hormones play a critical role in fetal development. Ramhoj et al. [18] studied the ef- fects of perfluorohexane sulfonate (PFHxS), a widely used environmental chemical found in breast milk, on fetal brain development. PFHxS has been proven to lower serum thyroxine in a rat model [19]. Treatment with PFHxS in early gestation lowered serum thyroxine in a rat model in both dams and their offspring but did not change thyroid-stimulating hormone levels. Additionally, there was no evidence of thyroid hormone-mediated neurobehavioral disruption in the offspring in that study. The authors pointed out that current rat models are not sensitive enough to detect neurodevelopmental deficits in early life. The developmental neurotoxicity of thyroid-disrupting chemicals (TDCs) and the current approaches designed to regulate them has also been recently reviewed [20].

Several novel TDCs were reported in 2020. Because of global restrictions on perfluorooctanesulfonic acid (PFOS) and perfluorooctanoic acid (PFOA), the use of long-chain perfluoroalkyl substances (PFASs, $>8$ carbon atoms) has increased. Kim et al. [21] found that long-chain perfluorinated carboxylic acids can cause transcriptional changes of thyroid-regulating genes that may lead to increased malformations of zebrafish larvae. The same group also demonstrated that 2-ethylhexyl-4-methoxycinnamate (EHMC), a widely used ultraviolet (UV) filter, induced hypothyroidism in association with neurotoxicity and nephrotoxicity [22]. Both studies needs to be validated with long-term exposure in adult animals.

\section{NOVEL THERAPEUTIC CHALLENGES IN THYROID CANCER}

Several steps were taken in 2020 towards solving unmet clinical needs in the treatment of advanced and metastatic thyroid cancers. Although radioactive iodine (RAI) therapy is considered to be effective for controlling metastatic lesions in differentiated thyroid cancer (DTC) patients, the survival rate markedly decreases when cancer starts the process of dedifferentiation, becoming RAI non-avid. Recently, studies have focused on re-differentiation therapy to restore iodine-avidity in cancer cells [23]. In the line with these efforts, Oh et al. [24] identified a novel tyrosine kinase inhibitor that restores RAI avidity in anaplastic thyroid cancer (ATC) cells by inhibiting both the mitogen-activated protein kinase (MAPK) and phosphatidylinositol 3-kinase (PI3K)/Akt signaling pathways. Another major challenge in the treatment of advanced thyroid cancer is how to control bone metastasis. Often, bone metastases in thyroid cancer are neglected in thyroid cancer patients; however, they are independently associated with poor prognosis with a median overall 
survival from detection of only 4 years despite an otherwise excellent prognosis for the vast majority of thyroid cancer patients [25]. Shin et al. [26] verified that metformin, which has been proven to have anti-cancer effects on thyroid cancer, effectively decreased tumor growth in a bone metastasis animal model by dual actions on bone and cancer cells. Interestingly, they showed that metformin modulates the microenvironment of the metastatic niche of bone by inhibiting the vicious cycle of cancer-bone interactions.

Two studies reported comprehensive immune profiling of thyroid cancers. Using fluorescent multiplex immunohistochemistry and multispectral imaging, Ahn et al. [27] conducted intensive immune profiling of advanced/metastatic DTC and ATC. They found that the density of programmed death-ligand 1 (PD-L1) and/or programmed death-1 (PD-1) positive lymphocytes was significantly higher in ATC than in metastatic DTCs. Another study was performed on medullary thyroid cancer (MTC) using immunohistochemistry and RNA sequencing to determine the immunogenic characteristics of MTC [28]. The authors concluded that MTC is a more immunologically active tumor that has been previously reported, and that further study is needed for immune therapy for MTC.

\section{CONCLUSIONS}

In 2020, basic and translational studies in thyroidology reported outstanding results. The studies discussed in this review have excellent clinical relevance and significance. Further bridging studies are needed to apply this knowledge in the clinical setting.

\section{CONFLICTS OF INTEREST}

No potential conflict of interest relevant to this article was reported.

\section{ORCID}

Sun Wook Cho https://orcid.org/0000-0002-7394-3830

Young Joo Park https://orcid.org/0000-0002-3671-6364

\section{REFERENCES}

1. Krieger TG, Moran CM, Frangini A, Visser WE, Schoenmakers E, Muntoni F, et al. Mutations in thyroid hormone receptor $\alpha 1$ cause premature neurogenesis and progenitor cell depletion in human cortical development. Proc Natl Acad Sci U S A 2019;116:22754-63.

2. Moran C, Schoenmakers N, Agostini M, Schoenmakers E, Offiah A, Kydd A, et al. An adult female with resistance to thyroid hormone mediated by defective thyroid hormone receptor $\alpha$. J Clin Endocrinol Metab 2013;98:4254-61.

3. Fauquier T, Romero E, Picou F, Chatonnet F, Nguyen XN, Quignodon L, et al. Severe impairment of cerebellum development in mice expressing a dominant-negative mutation inactivating thyroid hormone receptor alpha1 isoform. Dev Biol 2011;356:350-8.

4. Han CR, Wang H, Hoffmann V, Zerfas P, Kruhlak M, Cheng SY. Thyroid hormone receptor $\alpha$ mutations cause heart defects in zebrafish. Thyroid 2021;31:315-26.

5. Ferdous A, Wang ZV, Luo Y, Li DL, Luo X, Schiattarella GG, et al. FoxO1-Dio2 signaling axis governs cardiomyocyte thyroid hormone metabolism and hypertrophic growth. Nat Commun 2020;11:2551.

6. Franca MM, German A, Fernandes GW, Liao XH, Bianco AC, Refetoff S, et al. Human type 1 iodothyronine deiodinase (DIO1) mutations cause abnormal thyroid hormone metabolism. Thyroid 2021;31:202-7.

7. Kus A, Chaker L, Teumer A, Peeters RP, Medici M. The genetic basis of thyroid function: novel findings and new approaches. J Clin Endocrinol Metab 2020;105:dgz225.

8. Praestholm SM, Siersbaek MS, Nielsen R, Zhu X, Hollenberg AN, Cheng SY, et al. Multiple mechanisms regulate H3 acetylation of enhancers in response to thyroid hormone. PLoS Genet 2020;16:e1008770.

9. Coscia F, Taler-Vercic A, Chang VT, Sinn L, O'Reilly FJ, Izore $\mathrm{T}$, et al. The structure of human thyroglobulin. Nature 2020;578:627-30.

10. Knezevic J, Starchl C, Tmava Berisha A, Amrein K. Thyroid-gut-axis: how does the microbiota influence thyroid function? Nutrients 2020;12:1769.

11. Fenneman AC, Rampanelli E, Yin YS, Ames J, Blaser MJ, Fliers E, et al. Gut microbiota and metabolites in the pathogenesis of endocrine disease. Biochem Soc Trans 2020;48: 915-31.

12. Yoo WS, Chung HK. Recent advances in autoimmune thyroid diseases. Endocrinol Metab (Seoul) 2016;31:379-85.

13. Li Q, Wang B, Mu K, Zhang JA. The pathogenesis of thyroid autoimmune diseases: new $\mathrm{T}$ lymphocytes. Cytokines circuits beyond the Th1-Th2 paradigm. J Cell Physiol 2019;234:2204-16.

14. Su X, Yin X, Liu Y, Yan X, Zhang S, Wang X, et al. Gut 
dysbiosis contributes to the imbalance of treg and th17 cells in Graves' disease patients by propionic acid. J Clin Endocrinol Metab 2020;105:dgaa511.

15. Jiang W, Yu X, Kosik RO, Song Y, Qiao T, Tong J, et al. Gut microbiota may play a significant role in the pathogenesis of Graves' disease. Thyroid 2021 Jan 7 [Epub]. https:// doi.org/10.1089/thy.2020.0193.

16. Sun J, Zhao F, Lin B, Feng J, Wu X, Liu Y, et al. Gut microbiota participates in antithyroid drug induced liver injury through the lipopolysaccharide related signaling pathway. Front Pharmacol 2020;11:598170.

17. Lee DH. Evidence of the possible harm of endocrine-disrupting chemicals in humans: ongoing debates and key issues. Endocrinol Metab (Seoul) 2018;33:44-52.

18. Ramhoj L, Hass U, Gilbert ME, Wood C, Svingen T, Usai D, et al. Evaluating thyroid hormone disruption: investigations of long-term neurodevelopmental effects in rats after perinatal exposure to perfluorohexane sulfonate (PFHxS). Sci Rep 2020;10:2672.

19. Ramhoj L, Hass U, Boberg J, Scholze M, Christiansen S, Nielsen F, et al. Perfluorohexane sulfonate (PFHxS) and a mixture of endocrine disrupters reduce thyroxine levels and cause antiandrogenic effects in rats. Toxicol Sci 2018;163: 579-91.

20. Gilbert ME, O’Shaughnessy KL, Axelstad M. Regulation of thyroid-disrupting chemicals to protect the developing brain. Endocrinology 2020;161:bqaa106.

21. Kim J, Lee G, Lee YM, Zoh KD, Choi K. Thyroid disrupting effects of perfluoroundecanoic acid and perfluorotri- decanoic acid in zebrafish (Danio rerio) and rat pituitary (GH3) cell line. Chemosphere 2021;262:128012.

22. Chu S, Kwon BR, Lee YM, Zoh KD, Choi K. Effects of 2-ethylhexyl-4-methoxycinnamate (EHMC) on thyroid hormones and genes associated with thyroid, neurotoxic, and nephrotoxic responses in adult and larval zebrafish (Danio rerio). Chemosphere 2021;263:128176.

23. Pozdeyev N, Rose MM, Bowles DW, Schweppe RE. Molecular therapeutics for anaplastic thyroid cancer. Semin Cancer Biol 2020;61:23-9.

24. Oh JM, Baek SH, Gangadaran P, Hong CM, Rajendran RL, Lee HW, et al. A novel tyrosine kinase inhibitor can augment radioactive iodine uptake through endogenous sodium/ iodide symporter expression in anaplastic thyroid cancer. Thyroid 2020;30:501-18.

25. Iniguez-Ariza NM, Bible KC, Clarke BL. Bone metastases in thyroid cancer. J Bone Oncol 2020;21:100282.

26. Shin HS, Sun HJ, Whang YM, Park YJ, Park DJ, Cho SW. Metformin reduces thyroid cancer tumor growth in the metastatic niche of bone by inhibiting osteoblastic RANKL productions. Thyroid 2020 Sep 15 [Epub]. https://doi.org/10.1089/ thy.2019.0851.

27. Ahn J, Jin M, Song E, Ryu YM, Song DE, Kim SY, et al. Immune profiling of advanced thyroid cancers using fluorescent multiplex immunohistochemistry. Thyroid 2021;31: 61-7.

28. Pozdeyev N, Erickson TA, Zhang L, Ellison K, Rivard CJ, Sams S, et al. Comprehensive immune profiling of medullary thyroid cancer. Thyroid 2020;30:1263-79. 